\title{
A Numerical Method for Analysing Heat Conduction in Composites Containing Encapsulated Phase Change Materials
}

\author{
Hui Wang ${ }^{1}$ and Qing-Hua Qin ${ }^{2, a}$ \\ ${ }^{1}$ College of Civil Engineering \& Architecture, Henan University of Technology, Zhengzhou 450001, China \\ ${ }^{2}$ Research School of Engineering, Australian National University, Canberra, ACT 2601, Australia
}

\begin{abstract}
In this study, a three-dimensional transient heat transfer model in a three-phase composite system is established to investigate effects of temperature reduction in a composite system due to the use of encapsulated phase change material (PCM). The entire composite system is composed of cement matrix material, PCM, and hollow metal microspheres (HMSs) which are introduced to accelerate the phase change efficiency of the PCM and to simultaneously hold the liquid phase of the PCM. The present transient heat transfer model is numerically solved via finite element technique for investigating the transient thermal performance of the three-phase composite system. The temperature distribution on the specific area is compared to that in the pure cement material and the two-phase composite system without metal shell for demonstrating the ability of temperature adjustment of the PCM. Finally, effects of the spatial distribution of HMS on the temperature variation in the three-phase composite system is further investigated to provide comprehensive understanding on energy adjustment of this composite system.
\end{abstract}

\section{Introduction}

Efficient thermal energy controlling has received considerable attention in recent years in building industries. In doing so, the cement material, a type of most popular building material, is employed to couple with PCMs with high storage capacities for improving its performance of thermal energy storage. PCMs can store massive amount of latent heat at a constant temperature (phase change temperature of the PCM) during melting and solidification processes, and thus is very suitable for reducing energy consumption of buildings $[1,2]$.

However, PCMs usually have very low thermal conductivities, e.g. around $0.2 \mathrm{~W} /(\mathrm{mK})$ for octadecane and paraffin waxes and $0.5 \mathrm{~W} /(\mathrm{mK})$ for inorganic salts. This prolongs the charging and discharging period of latent heat, and causes slow thermal response in practical applications. To overcome this disadvantage of PCMs, different heat transfer enhancement technologies have been developed through adding different high thermal conductivity materials in cement composites, such as metal foams [3, 4], metal powders [5], and nanostructures [6-8], to increase their thermal conductivity. However, most of the existing studies based on the experimental research and the reported outcomes

\footnotetext{
${ }^{\mathrm{a}}$ Corresponding author: qinghua.qin@anu.edu.au
} 
are not consistent due to the limitations of experimental stuffs and approaches. Moreover, the mechanisms behind the changes of thermal properties need to be further explored.

Motivated from the discussion above, this report provides a three-dimensional transient thermal analysis of cement material filled with PCM enclosed inside hollow metal microspheres to examine the feasibility of temperature adjusting using encapsulated PCM in cement materials. In this work, the encapsulation of PCM is used as it can perfectly hold the liquid phase of the PCM and more importantly it has beneficial outcome on mechanical strength of building materials. Additionally, the thermal conductivity of solid metal phase is generally several thousand times higher than that of PCM, hence the usage of hollow metal microspheres can enhance the heat transfer capability of PCM inside them and accelerate the phase change efficiency of PCM. To demonstrate the effect of hollow metal microspheres on heat transfer enhancement in PCM system, the predicted results are compared to those without PCM or without hollow metal microspheres.

\section{Problem description}

The physical problem to be investigated is shown in Figure 1. The PCMs are encapsulated inside the hollow metal microspheres with outer diameter $d=50 \mathrm{~mm}$ and the thickness of the metal wall $t=5 \mathrm{~mm}$. They are, then, embedded into a cement material matrix. Five microspheres are arranged in a regular way as displayed in Figure 1. The distance between adjacent microspheres is denoted as $D$. Specially, when $D=2 R=d$, the adjacent microspheres are in contact each other. It is assumed that the composite system consisting of the PCM, metal spheres, and cement is heated from the top surface through a constant heat flux $G\left(\mathrm{Wm}^{-2}\right)$. The other surfaces are assumed perfectly insulated boundaries, as depicted in Figure 1.

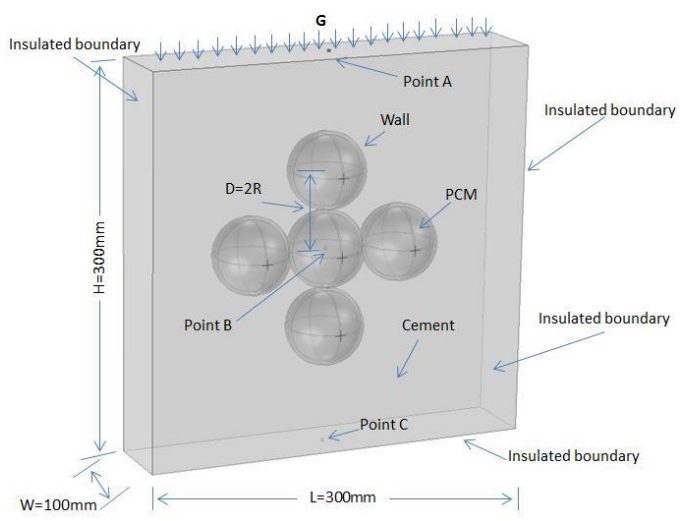

Figure 1: Illustration of cement composite with encapsulated PCM

In our computation, the octadecane is used as the PCM and the pure aluminium is chosen as the solid wall of the microspheres. The thermophysical properties of cement, aluminium and octadecane are tabulated in Table 1 for reference. Additionally, the initial conditions and the applied constant heat flux boundary condition are tabulated in Table 2 .

Table 1: Thermophysical properties of octadecane, aluminium wall and cement

\begin{tabular}{l|c|c|c}
\hline Property & Octadecane & Wall & Cement \\
\hline Density $\rho\left(\mathrm{kg} / \mathrm{m}^{3}\right)$ & 800 & 2675 & 1450 \\
\hline Mass heat capacity $c_{p}(\mathrm{~J} / \mathrm{kg} / \mathrm{k})$ & 1250 & 903 & 840 \\
\hline Latent heat of fusion $L_{f}(\mathrm{~J} / \mathrm{kg})$ & 125000 & $/$ & $/$ \\
\hline Melting temperature $T_{m}(\mathrm{~K})$ & 303 & $/$ & $/$ \\
\hline Thermal conductivity $k(\mathrm{~W} / \mathrm{m} / \mathrm{K})$ & 0.2 & 211 & 0.93 \\
\hline
\end{tabular}


Table 2: Initial and boundary conditions for the three-phase composite model

\begin{tabular}{c|c}
\hline Quantity & Value \\
\hline Initial temperature $T_{0}(K)$ & 293 \\
\hline Incident energy intensity $\mathrm{G}\left(\mathrm{Wm}^{-2}\right)$ & 750 \\
\hline
\end{tabular}

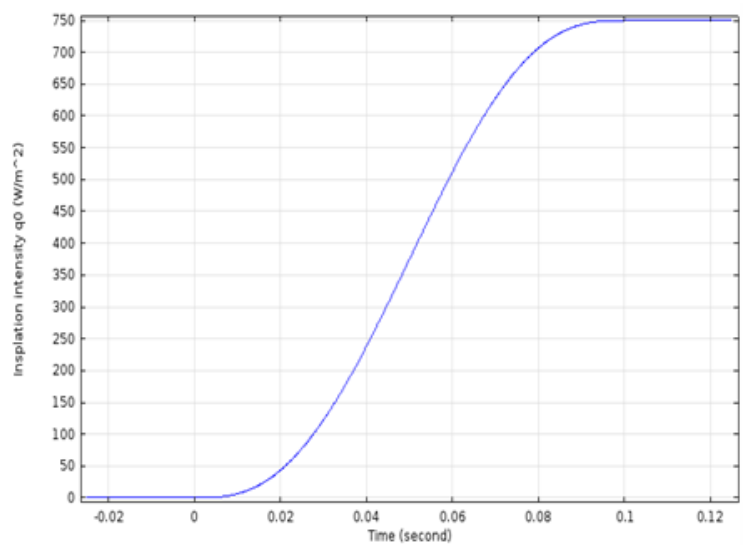

Figure 2: Smoothed incident energy intensity G

It is noted that the incident energy intensity, i.e. $G=750 \mathrm{Wm}^{-2}$, is applied on the top surface of the system since $t>0$. Obviously, it will cause a temperature discontinuity at the starting time. To fix this problem, a smoothed step function which increases the incident energy intensity from 0 to $750 \mathrm{Wm}^{-2}$ in $0.1 \mathrm{~s}$ is introduced, as shown in Figure 2.

\section{Numerical results and discuss}

In this section, the enhanced heat transfer in the present composite system is numerically investigated. Because the heat transfer in such multi-phase system is highly nonlinear, analytical solutions are only valid for some very simple cases [9-15]. Generally, numerical methods such as the finite element method [16-20], the boundary element method [21, 22], and the finite difference method [3, 23] are resorted to provide significant flexibility for achieving high quality of solutions and avoiding expensive experiments. Among them, the temperature-based finite element technique is very suitable for such phase-change problem involving moving melting boundary during the phase-change process and multiple materials considered in the paper. Hence, the finite element simulation is carried out through the commercial software COMSOL in this study. To show the effect of heat transfer enhancement induced by the hollow metal microspheres, the two-phase composite system including the cement material and the pure octadecane microspheres with same dimensions and the pure cement sample (without the PCM and the hollow metal microspheres) are also analyzed respectively for comparison. Additionally, three key points displayed in Figure 1 are chosen to investigate the temperature variation with the time. It is noted that Points $\mathrm{A}, \mathrm{B}$ and $\mathrm{C}$ are at the center of the top surface, the middle PCM microsphere, and the bottom surface, respectively.

In the following computation, the standard linear tetrahedron element is employed to model the pure cement sample, the two-phase composite system and the three-phase composite system, and the extra fine mesh scheme controlled by physical problem is used to guarantee the solution accuracy. The number of elements is 75351,239647 and 312195 for the three different systems, respectively, as shown in Figure 3. 

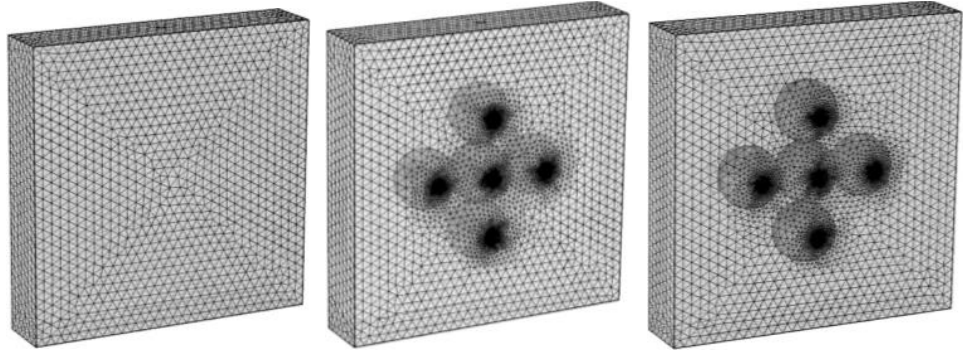

Figure 3. Mesh division for (a) the pure cement sample, (b) the two-phase composite system and (c) the threephase composite system

\subsection{Heat transfer in the pure cement}

Firstly, the pure cement sample is considered for investigating the temperature distribution in terms of time. From the numerical results shown in Figure 4, it is found that the temperature at Point A, which locates at the top heating surface, increases more quickly than those at Point B and C, which are far away from the heating surface. Because of the low thermal conductivity of cement material, the provided thermal energy reaches Point $\mathrm{B}$ and $\mathrm{C}$ at about 5100s and 17000s, respectively, and then causes temperature to increase. Before this, the temperature at Point $\mathrm{B}$ and $\mathrm{C}$ keeps in the initial temperature.

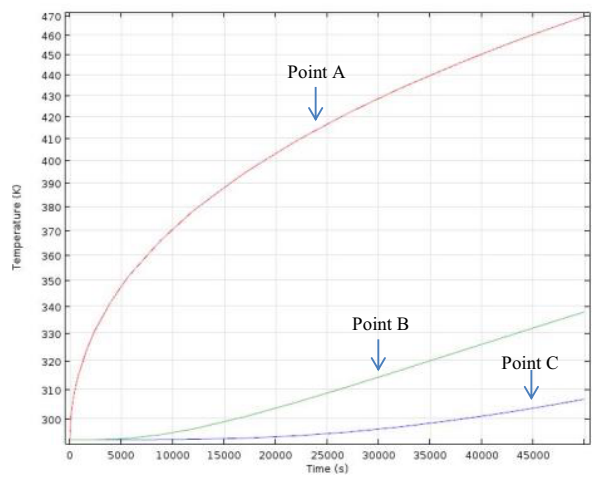

Figure 4. Temperature variation with time for the pure cement sample

\subsection{Heat transfer in the two-phase system}

Secondly, the heat transfer in the two-phase composite system consisting of the PCM microspheres and the cement material is considered. Results in Figure 5 indicate that the dramatic temperature increase at Point A is closely similar to that in the pure cement sample, whilst the temperature at Point $\mathrm{B}$ begins to slowly increase from the initial temperature at about $7200 \mathrm{~s}$, and it approximately reaches the melting temperature $303 \mathrm{~K}$ of the $\mathrm{PCM}$ at around $\mathrm{t}=44040 \mathrm{~s}$. This can be attributed to the presence of the PCM microsphere above the middle one. After this, the temperature at Point B keeps almost horizontal until 46440s. This means that the provided heat is used for overcoming the latent heat of fusion of the PCM around Point B to make it change its phase from the solid state to the liquid state and doesn't bring the temperature rise. After the PCM ball around Point B fully melts, the temperature starts to rise again due to the heat transfer in the fluid PCM. Compared to that in Figure 4 for the pure cement sample, however, the temperature variation at Point B in the PCM/CEMENT system is apparently slower. This can be attributed to the lower thermal conductivity of the PCM than that of the cement. Also, it is seen from Figure 5 that the rise of temperature at Point $\mathrm{C}$ occurs later, 
compared to the pure cement sample, as was expected. This can be attributed to the phase change of PCM balls. To observe the temperature variation in the system clearly, Figures 6(a), (b), (c) and (d) in which the green interface represents $303 \mathrm{~K}$, which is also the melting temperature of PCM, display the variation of temperature at different time instances. It is observed that the thermal energy transfers into all PCM balls very slowly, due to the lower thermal conductivity of the PCM than that of the cement. Besides, due to the presence of PCM, the isotherm becomes curved at the region near the PCM balls. The melting front surface of the PCM moves downward as the time increases and all PCM balls fully becomes liquid state at about 77640s, as shown in Figure 6(d) that the minimum temperature in the whole region is $303.403 \mathrm{~K}$.

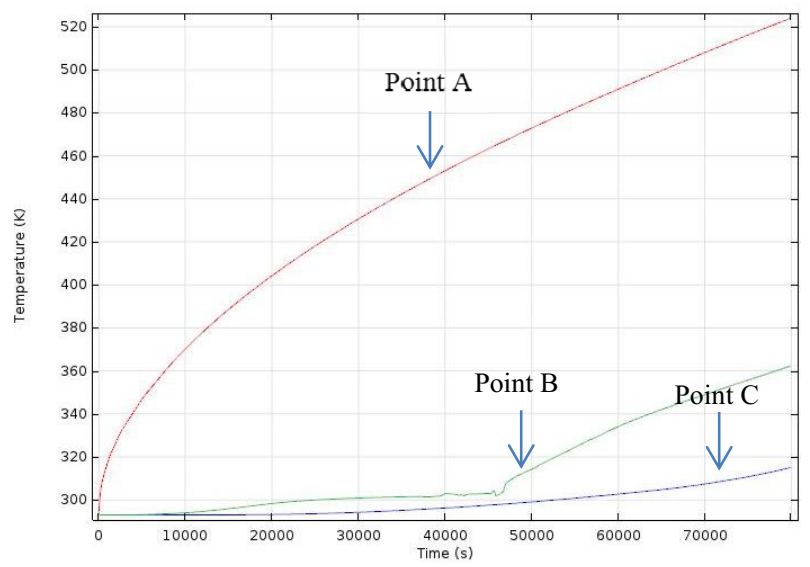

Figure 5: Temperature variation with time for the PCM/cement system

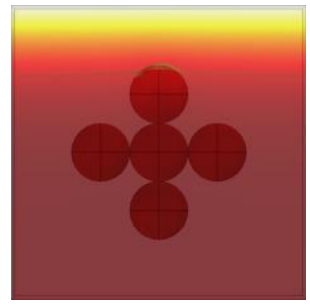

(a) $6600 \mathrm{~s}$

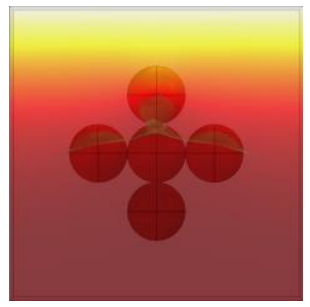

(b)22200s

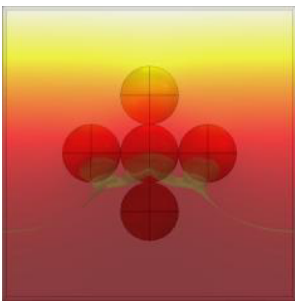

(c)44040s

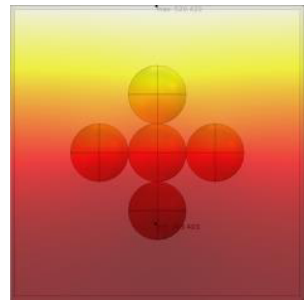

(d) $77640 \mathrm{~s}$

Figure 6: Temperature variation of the PCM/cement system at different time instances

\subsection{Heat transfer in the three-phase system}

Thirdly, the three-phase composite system is taken into consideration. Interestingly, as shown in figure 7, because the thermal conductivity of the aluminium wall is more than one thousand times higher than that of the PCM, the heat energy can rapidly transfer into the PCM and causes the temperature rise at point B quickly. At about 16980s, the temperature reaches the melting temperature and after this instance the temperature keeps unchanged due to the occurrence of phase change. Figures 8(a), (b), (c) and (d) clearly show the temperature variation with the time in the entire domain. It is clearly seen that all PCM balls encapsulated with aluminium spherical shell melt more rapidly than the pure PCM balls in the two-phase composite system, and all PCM balls fully becomes liquid state at about 43500 s. 


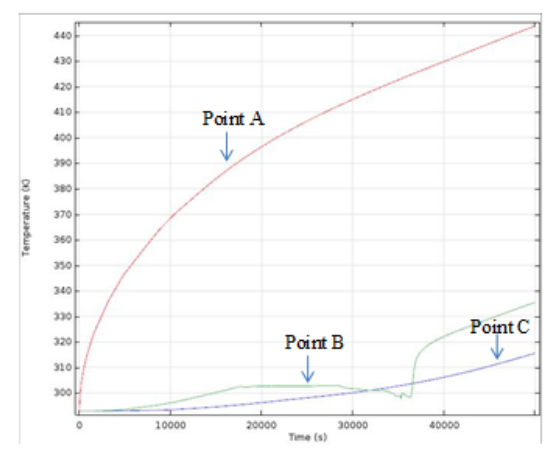

Figure 7: Temperature variation with time for the three-phase system

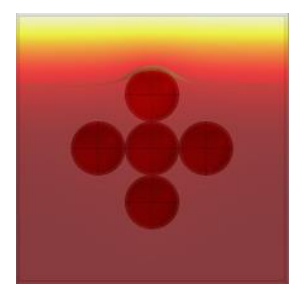

(a) $8100 \mathrm{~s}$

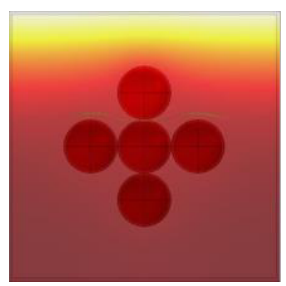

(b) $14100 \mathrm{~s}$

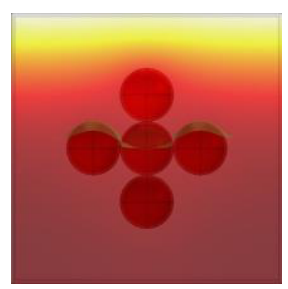

(c) $16980 \mathrm{~s}$

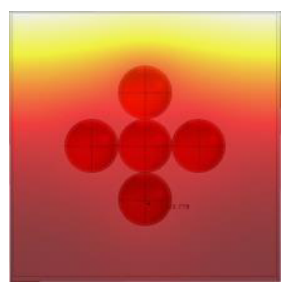

(d) $43500 \mathrm{~s}$

Figure 8: temperature variation of the three-phase system at different time instances

\subsection{Effect of the distribution of encapsulated PCMs}

From the computation above, it is found that the presence of hollow metal microsphere can improve the heat transfer efficiency in the PCM and leads to rapid phase change from the solid state to the liquid state. However, the adjacent PCM microspheres always keep in contact with each other in the above simulation. Actually, they can disperse in the cement material in any form. In order to investigate the effect of distribution of the five microspheres, a different three-phase composite model is considered, in which the four balls around the middle ball move outward by setting $D=150 \mathrm{~mm}$.

In Figure 9, the temperature platform at Point B appears at about 19680s which is evidently later than that in Figure 7. This is reasonable that the heating energy can't rapidly reach the middle metal microsphere embedded with the PCM because of the block of cement material between adjacent PCM microspheres. While for the model in Figure 7, the contact of adjacent metal microspheres makes the heating energy reach the middle metal microsphere more rapidly. This phenomenon can also be observed in Figure 10, which displays the temperature variation at different time instances. It is found that the large distance between neighboring encapsulated PCM microspheres alleviates the speed of thermal energy flowing into the PCM.

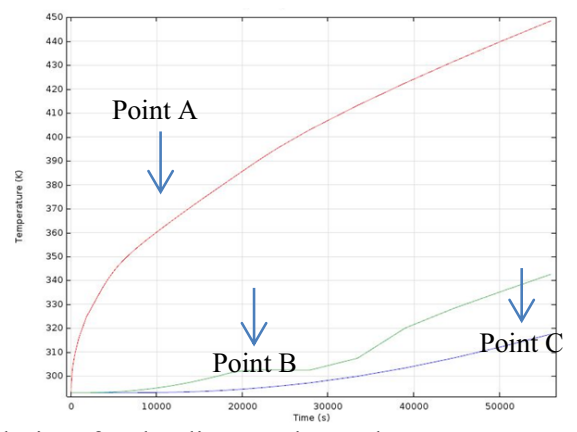

Figure 9: Temperature variation with time for the discrete three-phase system 


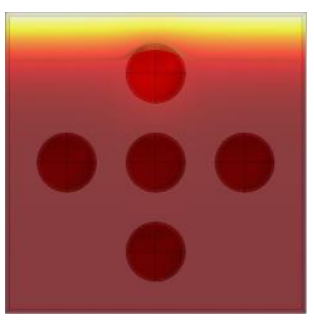

(a) $8100 \mathrm{~s}$

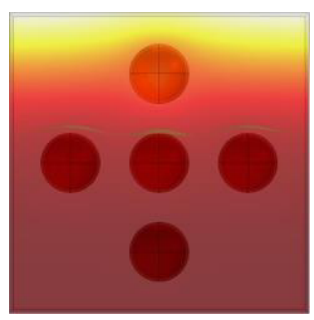

(b) $15000 \mathrm{~s}$

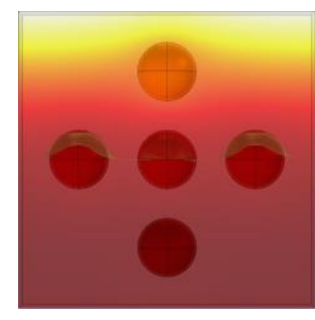

(c) $19680 \mathrm{~s}$

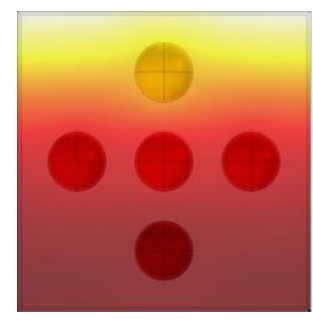

(d) $42000 \mathrm{~s}$

Figure 10: Temperature variation of the discrete three-phase at different time instances

\section{Conclusions}

In this study, the influence of heat transfer enhancement is numerically studied in three-phase composite system, in which the PCM are enclosed into the hollow metal microsphere and then are embedded into the cement matrix. Compared to the results from the pure cement sample, a two-phase system without the hollow metal microspheres, the effects of metal wall and distance between neighbouring PCM microspheres on solid/liquid phase change heat transfer are very significant. The introduction of hollow metal microsphere shortens the melting time of the whole system, as well as enhances the melting rate of PCM tremendously. Besides, the large distance between neighboring encapsulated PCM microspheres alleviates the speed of thermal energy flowing into the PCM system.

\section{References}

1. Zalba, B., J. M. Marin, L. F. Cabeza and H. Mehling, Applied Thermal Engineering, 23(3): 251283.(2003)

2. Zhou, D., C. Y. Zhao and Y. Tian, Applied Energy, 92: 593-605.(2012)

3. Zhao, C. Y., W. Lu and Y. Tian, Solar Energy, 84(8): 1402-1412.(2010)

4. Tian, Y. and C. Y. Zhao, Energy, 36(9): 5539-5546.(2011)

5. Mettawee, E. S. and G. M. R. Assassa, Solar Energy, 81(7): 839-845.(2007)

6. Xia, L., P. Zhang and R. Z. Wang, Carbon, 48(9): 2538-2548.(2010)

7. Ji, P., H. Sun, Y. Zhong and W. Feng, Chemical Engineering Science, 81: 140-145.(2012)

8. Şahan, N., M. Fois and H. O. Paksoy, Solar Energy Materials and Solar Cells, 137(137): 61-67.(2015)

9. Yu, S. and Q. H. Qin, Theoretical and Applied Fracture Mechanics, 25(3): 279-288.(1996)

10. Qin, Q. H. and S. W. Yu, International Journal of Solids and Structures, 34(5): 581-590.(1997)

11. Qin, Q. H., Y. W. Mai and S.-W. Yu, International Journal of Fracture, 91(4): 359-371.(1998)

12. Qin, Q. H. and Y. W. Mai, International Journal of Solids and Structures, 36(16): 2463-2479.(1999)

13. Qin, Q. H., Y. W. Mai and S. W. Yu, International journal of solids and structures, 36(3): 427439.(1999)

14. Qin, Q. H., International Journal of Solids and Structures, 37(39): 5561-5578.(2000)

15. Qin, Q. H. and J. Q. Ye, International Journal of Solids and Structures, 41(9-10): 2447-2460.(2004)

16. Dhanasekar, M., J. Han and Q. H. Qin, Finite Elements in Analysis and Design, 42(14-15): 13141323.(2006)

17. Qin, Q. H. and H. Wang (2008). Matlab and C programming for Trefftz finite element methods, CRC Press.

18. Wang, H. and Q. H. Qin, European Journal of Mechanics-A/Solids, 29(5): 801-809.(2010)

19. Wang, H. and Q. H. Qin, Archives of Mechanics, 62(6): 493-510.(2010)

20. Wang, H. and Q. H. Qin, Computational Mechanics, 48(5): 515-528.(2011)

21. Qin, Q. H., International journal of solids and structures, 30(22): 3101-3111.(1993)

22. Qin, Q. H. and Y. W. Mai, Engineering Fracture Mechanics, 69(5): 577-588.(2002)

23. Takeuchi, Y., Y. Yoshimoto and R. Suda, Journal of Computational and Applied Mathematics, 320: 101-119.(2017) 